\title{
Clinical and radiographic evaluation of jumping distance management using a collagen matrix in flapless immediate implant placement
}

\author{
Mahdi Kadkhodazadeh ${ }^{1,2, A, B, D, F}$, Reza Amid ${ }^{1,2, C, E, F}$, Anahita Moscowchi ${ }^{2, C-E}$, Vahid Khoshkam ${ }^{3, C-E}$ \\ ${ }^{1}$ Research Institute for Dental Sciences, Shahid Beheshti University of Medical Sciences, Tehran, Iran \\ ${ }^{2}$ Department of Periodontics, Shahid Beheshti University of Medical Sciences, Tehran, Iran \\ 3 Private practice, El Paso, USA \\ A - research concept and design; B - collection and/or assembly of data; $\mathrm{C}$ - data analysis and interpretation; \\ $D$ - writing the article; $E$ - critical revision of the article; $F$ - final approval of the article
}

Address for correspondence

Anahita Moscowchi

E-mail: a.moscowchi@gmail.com

Funding sources

None declared

Conflict of interest

None declared

Received on May 11,2020

Reviewed on August 30, 2020

Accepted on November 4, 2020

Published online on May 11, 2021

\begin{abstract}
Background. Improvement in implant design has made implant dentistry a challenging treatment modality worldwide.

Objectives. This study aimed to investigate the efficacy of a xenogeneic collagen matrix in managing 3-4-millimeter gaps in flapless immediate implant placement.
\end{abstract}

Material and methods. Twenty-two patients received 39 immediate implants via the flapless approach. Patients with intact bony walls, buccal bone thickness $\geq 2 \mathrm{~mm}$ and a jumping distance of $3-4 \mathrm{~mm}$ were included in this study. The gap between the implant and the socket walls was filled with a xenogeneic collagen matrix (Collacone $\left.{ }^{\circledR}\right)$. The final clinical and radiographic evaluations were performed at least 24 months following functional loading.

Results. There was no early or late failure, and the implants showed a 100\% survival rate. The mean mesial $(0.28 \pm 0.39 \mathrm{~mm})$ and distal $(0.28 \pm 0.39 \mathrm{~mm})$ marginal bone loss (MBL) at the site of incisors was not significantly different from the values at the site of premolars and molars $(0.30 \pm 0.42 \mathrm{~mm}$ and $0.34 \pm 0.48 \mathrm{~mm}$, respectively). The evaluation of the implant success index (ISI) score revealed no difference between the mandible and the maxilla $(p=0.700)$, or incisors compared with premolars and molars $(p=0.420)$. The only significant difference was in terms of distal MBL, which was higher in the maxilla $(0.39 \pm 0.49 \mathrm{~mm})$ than in the mandible $(0.12 \pm 0.23 \mathrm{~mm})(p=0.040)$.

Conclusions. Within the limitations of this study, it seems that the application of a xenogeneic collagen matrix to manage 3-4-millimeter gaps in carefully selected cases may bring promising outcomes.

Keywords: peri-implantitis, alveolar bone loss, collagen matrix, immediate dental implant

Cite as

Kadkhodazadeh M, Amid R, Moscowchi A, Khoshkam V. Clinical and radiographic evaluation of jumping distance management using a collagen matrix in flapless immediate implant placement. Dent Med Probl. 2021;58(2):173-178. doi:10.17219/dmp/130057

DOI

$10.17219 / \mathrm{dmp} / 130057$

Copyright

๑ 2021 by Wroclaw Medical University

This is an article distributed under the terms of the

Creative Commons Attribution 3.0 Unported License (CC BY 3.0)

(https://creativecommons.org/licenses/by/3.0/). 


\section{Introduction}

The use of dental implants to replace the missing teeth was a great scientific breakthrough in the field of dentistry. Implant dentistry has evolved considerably, as the original protocol was modified to adopt one-stage surgery ${ }^{1}$ and immediate implant placement protocols. ${ }^{2}$ Although there have been some reports of peri-implantitis and implant failure, immediate dental implants can offer a success/survival rate comparable to implants placed with early and delayed protocols, as long as the existing guidelines are strictly followed. ${ }^{3}$

It has been well documented that implant placement in a fresh extraction socket does not counteract bone remodeling. Therefore, both vertical and horizontal dimensional changes may be expected. ${ }^{4}$ Several factors affect the outcome of immediate implantation, such as the flap or flapless approach, ${ }^{5}$ the thickness of the buccal bone, ${ }^{6}$ the gingival biotype, the timing of restoration placement, ${ }^{7}$ the distance from the implant platform to the crestal bone, implant surface topography, and the size of the gap between the implant and the alveolar socket walls. ${ }^{6,8}$

The distance between the socket walls and the implant surface may justify augmentation to predictably achieve bone-implant contact (BIC) and to prevent soft tissue collapse. ${ }^{9}$ However, there has been controversy in the literature in this regard. Some preclinical and clinical studies have documented the regeneration of horizontal gaps smaller than $2 \mathrm{~mm}$ in the presence of a stable blood clot. ${ }^{10,11}$ More recent studies have shown that gaps of more than $2 \mathrm{~mm}$ have a tendency to fill better, even without grafting material. ${ }^{8}$ A previous study compared flap and flapless surgery for immediate implantation, and concluded that the flapless approach allowed for minimal surgical intervention with comparable bony changes and gap filling after a 6-month follow-up in areas with sufficient buccal bone support. ${ }^{12}$

Bone formation in jumping distances of various sizes remains a controversial topic. Moreover, there have been few investigations on the secondary healing of the implant-buccal bone interface, and the current evidence referring to postextraction alveolar bone alterations in regard to the thickness of the buccal plate remains unclear. However, some studies have shown that the thickness of the buccal plate and the size of the jumping distance play critical roles in the filling of the gap. ${ }^{6}$ This study assessed the clinical and radiographic outcomes of immediate implant placement with the use of a xenogeneic collagen matrix to fill the jumping distance in cases with a thick buccal plate ( $\geq 2 \mathrm{~mm}$ ).

\section{Material and methods}

This study was conducted from September 2016 to February 2020 at the Department of Periodontics, Shahid Beheshti University of Medical Sciences, Tehran, Iran, in accordance with the Declaration of Helsinki for human studies. It was approved by the Ethics Committee of Shahid Beheshti University of Medical Sciences (IR.SBMU.DRC.REC.1398.164), and written informed consent was obtained from all patients prior to their enrollment.

\section{Study population}

Twenty-two patients (11 males and 11 females) were included in this study. The inclusion criteria were as follows: patients between 20 and 40 years of age; having a hopeless tooth indicated for extraction (due to an unrestorable fracture or a carious lesion) with no active infection at the surgical site; at least a 4-millimeter distance between the tooth apex and the maxillary sinus floor; the absence of gingival inflammation; achieving adequate primary stability following immediate implant placement; buccal bone thickness $\geq 2 \mathrm{~mm}$, measured using the NNT software (NewTom Company, Imola, Italy); intact socket walls following the extraction; gingival thickness $\geq 1.5 \mathrm{~mm}$, measured using a periodontal probe; presenting for a visit after at least 24 months of functional loading; O'Leary's plaque index $\leq 20 \%$; and class I dental socket based on a conebeam computed tomography (CBCT) scan. ${ }^{13}$ The exclusion criteria were as follows: poor oral hygiene or the lack of regular maintenance; compromised immune system, systemic diseases or an intake of medications; the use of orthodontic appliances; the presence of parafunctional habits (bruxism or clenching); external root resorption; a jumping distance $>4 \mathrm{~mm}$, measured using a periodontal probe; smoking; and anatomical limitations.

\section{Study overview and interventions}

All patients received phase I periodontal therapy and their hard tissue condition was evaluated preoperatively with CBCT. The patients rinsed their mouths with $0.2 \%$ chlorhexidine for $1 \mathrm{~min}$ right before local anesthesia administration (20 mg/mL lidocaine with 1:80,000 epinephrine). All steps were performed conservatively by an experienced periodontist (M.K.), with no soft tissue manipulation, using the flapless approach. Following tooth extraction, the socket walls were examined with a periodontal probe, and in case of fenestration or dehiscence, the patient was excluded from the study. The sockets were cleaned using a curette and rinsed with saline. Drilling was performed based on the manufacturer's instructions with palatal orientation and 2-3 mm apical to the extraction socket, according to the selected implant system: SPI ${ }^{\circledR}$ (Thommen Medical, Grenchen, Switzerland); SIC $^{\circledR}$ (SIC invent, Basel, Switzerland); 3i (Zimmer Biomet, Warsaw, USA); BioHorizons (BioHorizons Implant Systems, Birmingham, USA); Euroteknika (Euroteknika, Sallanches, France); or Intra-Lock ${ }^{\circledR}$ (Intra-Lock International, Boca Raton, USA) (Table 1). Implants of different root forms were selected based on the implant site and bone condition. The implants were inserted $1 \mathrm{~mm}$ apical to the alveolar crest at 30-35 N/cm. A xenogeneic collagen matrix (Collacone ${ }^{\circledR}$; Botiss Biomaterials, Zossen, Germany) was then applied into the jumping distance (Fig. 1). No sutures or other materials 
Table 1. Patient information, implant data and the implant success index (ISI) scores

\begin{tabular}{|l|c|}
\hline Characteristic & Description \\
\hline Patient gender $(N=22)$ & female $(n=11)$ \\
& male $(n=11)$ \\
& SPI $(n=13)$ \\
& SIC $(n=2)$ \\
& 3 i $(n=3)$ \\
Implant system $(N=39)$ & BioHorizons $(n=2)$ \\
& Euroteknika $(n=8)$ \\
Intra-Lock $(n=11)$ \\
Implant location $(N=39) \quad$ maxillary incisors $(n=7)$ \\
maxillary premolars $(n=18)$ \\
maxillary molars $(n=6)$ \\
mandibular premolars $(n=5)$ \\
mandibular molars $(n=3)$ \\
Implant parameters \\
diameter: $3.3-5$ mm \\
length: $8-14$ mm
\end{tabular}

The implant success index (ISI) according to Kadkhodazadeh M, Amid R. Evaluation of peri-implant tissue health using a scoring system. JIACD. 2012;4(1):51-57 (cf. Table 2).

were applied to close the socket or to cover it. The healing abutment was screwed into all implants so that it was $2 \mathrm{~mm}$ coronal to the gingival margin. None of the implants were immediately restored.

The patients and their companions were provided with postoperative instructions, which included rinsing with a chlorhexidine mouthwash 3 times a day, $400 \mathrm{mg}$ ibuprofen every $6 \mathrm{~h}$, and $500 \mathrm{mg}$ amoxicillin every $8 \mathrm{~h}$ for 1 week or an alternative in case of being allergic to penicillin. The patients were followed up weekly in the $1^{\text {st }}$ month and monthly during the next 3 months. All final restorations were provided by the same prosthodontist and dental laboratory (after 3 months in the mandible and after 4 months in the maxilla).

The recall program was scheduled at 6-month intervals. ${ }^{14}$ After at least 24 months of functional loading, the patients were recalled for clinical and radiographic examinations (Fig. 2). Periapical radiographs (the parallel technique) were
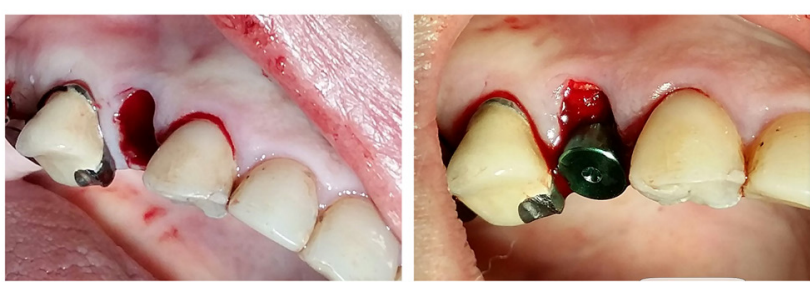

Fig. 1. Atraumatic tooth extraction performed without flap reflection and the application of a xenogeneic collagen matrix (Collacone $\left.{ }^{\circledR}\right)$ into the gap between the socket wall and the implant surface following implant insertion

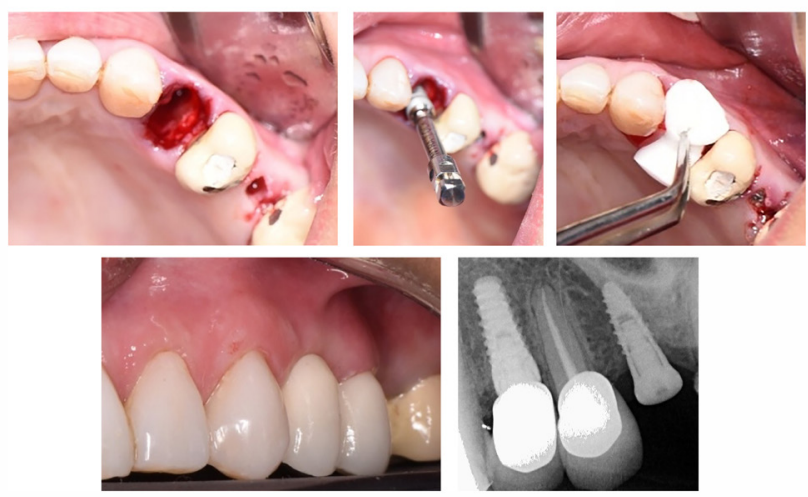

Fig. 2. Immediate implant placement with the use of a xenogeneic collagen matrix; 2-year clinical and radiographic follow-up demonstrates a well-shaped soft tissue contour and a stable marginal bone level

obtained to assess the implants, the restorations and the surrounding tissues. Marginal bone loss (MBL) was measured relative to the implant shoulder with the use of digital software (Scanora ${ }^{\mathrm{TM}}$; KaVo, Biberach, Germany) and the probing depth (PD) was measured using a plastic periodontal probe. Next, the pink esthetic score (PES) was determined for all implants. ${ }^{15}$ Clinical measurements were made at 4 points around each implant by an expert periodontist (M.K.) with an intra-rater agreement of 0.93 based on the intraclass correlation coefficient (ICC). Oral hygiene instructions were regularly reinforced at each session and professional cleaning was performed as required. All implants were evaluated based on the implant success index (ISI) proposed by Kadkhodazadeh and Amid (Table 2). ${ }^{16}$

Table 2. Implant success index (ISI)

\begin{tabular}{|c|c|c|c|}
\hline Score & SL & HL & Clinical findings \\
\hline$|S| \mid$ & $\mathrm{SL}+, \mathrm{PPD} \leq 4 \mathrm{~mm}, \mathrm{BOP}-$ & $\mathrm{HL}+$ & clinically healthy \\
\hline ISI II & $\mathrm{SL}+, \mathrm{PPD} \leq 4 \mathrm{~mm}, \mathrm{BOP}+$ & $\mathrm{HL}+$ & soft tissue inflammation \\
\hline$|S|$ III & $\mathrm{SL}+, \mathrm{PPD}>4 \mathrm{~mm}, \mathrm{BOP}+$ & $\mathrm{HL}+$ & deep soft tissue pocket \\
\hline ISI IV & $\mathrm{SL}+$ & $\mathrm{HL}-, \mathrm{RBL} \leq 2 \mathrm{~mm}(\leq 20 \%)$ & initiation of hard tissue breakdown \\
\hline ISIV & $\mathrm{SL}-$ & $\mathrm{HL}-, \mathrm{RBL} \leq 2 \mathrm{~mm}(\leq 20 \%)$ & hard tissue breakdown and soft tissue recession \\
\hline ISI VI & $\mathrm{SL}+$ & $\mathrm{HL}-, \mathrm{RBL}: 2-4 \mathrm{~mm}(<40 \%)$ & notable hard tissue breakdown \\
\hline ISI VII & $\mathrm{SL}-$ & $\mathrm{HL}-, \mathrm{RBL}: 2-4 \mathrm{~mm}(<40 \%)$ & notable hard tissue breakdown and soft tissue recession \\
\hline ISI VIII & - & $\mathrm{RBL} \geq 40 \%$ & severe bone loss \\
\hline ISI IX & - & clinical mobility & clinical failure \\
\hline
\end{tabular}

SL - soft tissue level; HL - hard tissue level; PPD - probing pocket depth; BOP - bleeding on probing; RBL - radiographic bone loss detected via the long cone paralleling peri-apical technique; + tissue level located at or coronal to the reference line; - tissue level located apical to the reference line; if the peri-apical area of implants has a bone loss/radiolucent view (retrograde peri-implantitis), it is identified by placing the letter R (e.g., ISI IR, etc.). The Table is adapted from: Kadkhodazadeh M, Amid R. Evaluation of peri-implant tissue health using a scoring system. JIACD. 2012;4(1):51-57. 


\section{Statistical analyses}

Data analyses were conducted using IBM SPSS Statistics for Windows, v. 22 (IBM Corp., Armonk, USA). The ISI scores were compared using the $X^{2}$ test. The independent $t$ tests were used to analyze the mean PD, MBL and PES values. A $p$-value $<0.05$ was considered statistically significant with a $95 \%$ confidence interval $(C I)$.

\section{Results}

All the included patients were followed up for a period of 24-72 months. During the study period, all surgical sites healed uneventfully with no signs of gingival inflammation, infection or esthetic complications.

Table 3 presents $\mathrm{PD}$, radiographic MBL as well as PES with regard to implant location. The PD and MBL scores at the site of incisors were compared with the corresponding values at the site of premolars and molars. The mean buccal PD was $2.29 \pm 0.49 \mathrm{~mm}$ at the site of incisors, and $2.25 \pm 0.44 \mathrm{~mm}$ at the site of premolars and molars. No significant difference was observed between the 2 groups $(p=0.850)$. The mean lingual PD at the site of incisors $(2.14 \pm 0.38 \mathrm{~mm})$ was not significantly different from the value measured at the site of premolars and molars $(2.21 \pm 0.55 \mathrm{~mm})(p=0.860)$. The mean PD was not significantly different in the maxilla and the mandible $(p=0.850$ for buccal PD and $p=0.890$ for lingual PD).

Marginal bone loss was also evaluated at different implant locations and for both jaws. The mean mesial MBL was $0.28 \pm 0.39 \mathrm{~mm}$ at the site of incisors, and $0.30 \pm 0.42 \mathrm{~mm}$ at the site of premolars and molars, which showed no significant difference $(p=0.950)$. There was no significant difference in the mean distal MBL at the site of incisors $(0.28 \pm 0.39 \mathrm{~mm})$ in comparison with premolars and molars $(0.34 \pm 0.48 \mathrm{~mm})(p=0.770)$. The mesial MBL was not significantly different in the maxilla and the mandible $(p=0.070)$; however, there was a significant difference in the distal MBL $(p=0.040)$, which was $0.39 \pm 0.49 \mathrm{~mm}$ in the maxilla and $0.12 \pm 0.23 \mathrm{~mm}$ in the mandible. The PES was also determined for each implant, and it showed no significant difference at different sites $(p=0.150)$ or jaws $(p=0.540)$.

The results of the ISI score evaluation revealed that there was no significant difference between the mandible and the maxilla ( $p=0.700)$, and incisors compared with premolars and molars $(p=0.420)$.

\section{Discussion}

Although fewer surgical procedures, optimal costeffectiveness and a shorter treatment time have made immediate implantation a desirable option, the inability to predict soft and hard tissue levels, difficulty in achieving primary stability, and the presence of the jumping distance intervening with direct BIC may jeopardize a favorable outcome. ${ }^{17}$ Different biomaterials, including autogenous, allogenic and xenogeneic grafts, have been proposed in the literature to successfully fill the jumping distance. ${ }^{18-20}$ However, there is concern that the residual particles may interfere with efficient BIC. In the present study, the aim was to find an alternative to biomaterials for 3-4-millimeter gaps; for this purpose, a xenogeneic collagen matrix was used to preserve the blood clot and prevent soft tissue collapse

Table 3. Probing depth (PD), marginal bone loss (MBL) and the pink esthetic score (PES) at different implant locations after at least 24 months of functional loading

\begin{tabular}{|c|c|c|c|c|c|}
\hline \multirow{2}{*}{ Dependent variable } & \multicolumn{5}{|c|}{ Implant location } \\
\hline & $\begin{array}{l}\text { incisors } \\
(n=7)\end{array}$ & $\begin{array}{c}\text { premolars } \\
(n=23)\end{array}$ & $\begin{array}{l}\text { molars } \\
(n=9)\end{array}$ & $\begin{array}{l}\text { maxilla } \\
(n=31)\end{array}$ & $\begin{array}{c}\text { mandible } \\
(n=8)\end{array}$ \\
\hline $\begin{array}{l}\text { PD buccal } \\
\text { [mm] } \\
p \text {-value }\end{array}$ & $2.29 \pm 0.49$ & $\begin{array}{c}2.21 \pm 0.42 \\
0.850\end{array}$ & $2.33 \pm 0.50$ & $2.26 \pm 0.44$ & $2.25 \pm 0.57$ \\
\hline $\begin{array}{c}\text { PD lingual/platal } \\
\text { [mm] } \\
p \text {-value }\end{array}$ & $2.14 \pm 0.38$ & $\begin{array}{c}2.26 \pm 0.54 \\
0.860\end{array}$ & $2.11 \pm 0.60$ & $2.26 \pm 0.57$ & $2.20 \pm 0.00$ \\
\hline $\begin{array}{l}\text { MBL mesial } \\
{[\mathrm{mm}]} \\
p \text {-value }\end{array}$ & $0.28 \pm 0.39$ & $\begin{array}{c}0.23 \pm 0.33 \\
0.950\end{array}$ & $0.44 \pm 0.58$ & $0.33 \pm 0.43$ & $0.12 \pm 0.23$ \\
\hline $\begin{array}{c}\text { MBL distal } \\
\text { [mm] } \\
p \text {-value }\end{array}$ & $0.28 \pm 0.39$ & $\begin{array}{c}0.28 \pm 0.42 \\
0.770\end{array}$ & $0.50 \pm 0.61$ & $0.39 \pm 0.49$ & $0.12 \pm 0.23$ \\
\hline $\begin{array}{c}\text { PES } \\
p \text {-value }\end{array}$ & $9.86 \pm 0.38$ & $\begin{array}{c}9.52 \pm 0.59 \\
0.150\end{array}$ & $9.78 \pm 0.44$ & $9.68 \pm 0.47$ & $9.50 \pm 0.76$ \\
\hline
\end{tabular}

${ }^{*}$ statistically significant.

Data presented as mean $(M) \pm$ standard deviation $(S D)$ 
(although slightly). Collacone is a moldable xenogeneic collagen matrix designed to support the natural healing of extraction sockets. Its application supports the stabilization of the formed blood clot, helps to control bleeding, and protects the wound area from food residue and bacteria. Collacone resorbs completely in about 2-4 weeks with no remnants; thus, it does not seem to interfere with BIC.

After at least 24 months of functional loading, all implants were osseointegrated with minimal bone loss. As all restorations were cement-retained, the measurements were conducted with the presence of the restorations. The PD scores were within the normal range and were similar to those reported in other studies in which implants were placed immediately or delayed..$^{21}$

Marginal bone loss is among the most significant variables, as it may predispose implants to peri-implantitis and result in implant failure. ${ }^{22}$ Since immediate implants are often placed apical to the alveolar crest, some researchers have reported that they may compromise accurate bone level measurements. As previously emphasized, the available supporting bone is an important parameter to consider. ${ }^{23}$ In this study, bone loss relative to the implant shoulder was $\leq 1 \mathrm{~mm}$ (the mesial MBL was $0.28 \pm 0.39 \mathrm{~mm}$ at the site of incisors, and $0.30 \pm 0.42 \mathrm{~mm}$ at the site of premolars and molars, while the distal MBL was $0.28 \pm 0.39 \mathrm{~mm}$ at the site of incisors compared with $0.34 \pm 0.48 \mathrm{~mm}$ at the site of premolars and molars). Although there was a difference in terms of distal MBL between the maxilla and the mandible, it did not seem to be clinically significant. In addition, the high standard deviation $(S D)$ values mean that the numbers are more spread out. Therefore, further studies with larger sample sizes are advocated to avoid errors from the testing of possibly atypical samples. More importantly, most previous studies, as well as the present one, which examined MBL with the use of the parallel radiographic technique evaluated only the mesial and distal surfaces; however, significant changes may also occur at the buccal surface in the long term, which may adversely affect the esthetic outcome. ${ }^{22}$ Nevertheless, considering our strict inclusion criteria (buccal bone thickness $\geq 2 \mathrm{~mm}$ ), we concluded that there was no need to evaluate the buccal bone by means of three-dimensional (3D) radiography during follow-up visits. Furthermore, we included teeth with a thick gingival biotype, which has been shown to improve soft and hard tissue stability in immediate implantation. ${ }^{24}$

Soft tissue considerations, specifically midfacial recession, is another important issue regarding immediate implant placement. ${ }^{17}$ Peri-implant soft tissue management can influence implant success and prevent peri-implantitis. It has been documented that adequately keratinized gingiva around implants is especially important in the esthetic zone to provide a more natural soft tissue drape. In the case of adopting the non-submerged protocol, the use of xenogeneic collagen materials such as Collacone may resolve the concern.
Socket closure and soft tissue management are among other controversial topics in immediate implant placement. Attempts to stabilize the peri-implant tissue after tooth extraction and immediate implant placement include a variety of wound closure techniques that use different types of flaps. Tarnow et al. evaluated changes in the facial and palatal ridges during flapless immediate implant placement and showed less bone resorption with the use of bone graft material along with a provisional restoration, ${ }^{25}$ even though a recent study showed that the placement of an immediate provisional restoration would not significantly improve the esthetics. ${ }^{7}$

The absence of infection at the site of immediate implant placement is another critical factor that affects long-term success and prevents postoperative complications. ${ }^{26}$ Some studies have reported retrograde periimplantitis following immediate implant placement in the extraction sockets of teeth with periapical lesions. ${ }^{27}$ However, meticulous socket debridement may decrease the risk of retrograde peri-implantitis. Furthermore, the type of dental socket, and the proper selection of the diameter and length of the implant are of paramount importance in implant stability. ${ }^{28}$

Another noteworthy issue is that proper oral hygiene and regular recall visits maintain the treatment results and prevent adverse consequences. Therefore, this study included patients with adequate plaque control, who followed a strict oral hygiene routine and attended follow-up assessments. ${ }^{29}$

The favorable results of the current study might be partially related to the applied flapless technique for immediate implant placement. However, the available evidence in the literature is not yet conclusive regarding the superiority of performing immediate implant placement in a flapless manner. ${ }^{5}$

The thickness of the buccal plate and the size of the gap have been shown to be the most influential factors in gap filling. ${ }^{8}$ In a randomized clinical trial, it was shown that the thickness of the buccal plate was the most determinant factor in the resorption of the alveolar bone following extraction ${ }^{30}$; hence, randomized clinical trials are needed to compare the method applied in the present study with spontaneous healing in cases with a buccal bone thickness of more than $2 \mathrm{~mm}$. Also, the effect of filling the gap in sockets with thinner buccal plates needs further investigation.

\section{Limitations}

As with any investigation, the present study has a number of limitations. The analyzed radiographs were not standardized, which potentially might have led to measurement errors. However, the calibration of radiographs by using known implant dimensions minimized this limitation. 


\section{Conclusions}

Implant success/failure is the most frequently reported outcome measure in the literature; however, it is more favorable to evaluate and compare peri-implant health and disease condition, as discussed in the proposed ISI. ${ }^{16}$ Our results showed a $100 \%$ success rate, healthy surrounding tissues and minimal bone level alterations for immediate implants placed in sockets with buccal plates $\geq 2 \mathrm{~mm}$. Despite the limitations of this study, it seems that the application of a xenogeneic collagen matrix without bone grafting particles may provide promising outcomes in the management of 3-4-millimeter jumping distances in carefully selected cases.

\section{ORCID iDs}

Mahdi Kadkhodazadeh (D) https://orcid.org/0000-0002-6131-2791 Reza Amid (D) https://orcid.org/0000-0002-8053-3928 Anahita Moscowchi (D) https://orcid.org/0000-0003-1327-5014 Vahid Khoshkam (10) https://orcid.org/0000-0002-6405-1910

\section{References}

1. Becker W, Becker BE, Israelson $\mathrm{H}$, et al. One-step surgical placement of Brånemark implants: A prospective multicenter clinical study. Int J Oral Maxillofac Implants. 1997;12(4):454-462.

2. Lazzara RJ. Immediate implant placement into extraction sites: Surgical and restorative advantages. Int J Periodontics Restorative Dent. 1989;9(5):332-343.

3. Hayacibara RM, Gonçalves CS, Garcez-Filho J, Magro-Filho O, Esper H, Hayacibara MF. The success rate of immediate implant placement of mandibular molars: A clinical and radiographic retrospective evaluation between 2 and 8 years. Clin Oral Implants Res. 2013;24(7):806-811. doi:10.1111/j.1600-0501.2012.02461.x

4. Araújo MG, Sukekava F, Wennström JL, Lindhe J. Tissue modeling following implant placement in fresh extraction sockets. Clin Oral Implants Res. 2006;17(6):615-624. doi:10.1111/j.1600-0501.2006.01317.x

5. Caneva M, Botticelli D, Salata LA, Scombatti Souza SL, Bressan E, Lang NP. Flap vs. "flapless" surgical approach at immediate implants: A histomorphometric study in dogs. Clin Oral Implants Res. 2010;21(12):1314-1319. doi:10.1111/j.1600-0501.2010.01959.x

6. Ferrus J, Cecchinato D, Pjetursson EB, Lang NP, Sanz M, Lindhe J. Factors influencing ridge alterations following immediate implant placement into extraction sockets. Clin Oral Implants Res. 2010;21(1):22-29. doi:10.1111/j.1600-0501.2009.01825.x

7. Chan HL, George F, Wang IC, López Del Amo FS, Kinney J, Wang HL. A randomized controlled trial to compare aesthetic outcomes of immediately placed implants with and without immediate provisionalization. J Clin Periodontol. 2019;46(10):1061-1069. doi:10.1111/jcpe.13171

8. Sanz M, Lindhe J, Alcaraz J, Sanz-Sanchez I, Cecchinato D. The effect of placing a bone replacement graft in the gap at immediately placed implants: A randomized clinical trial. Clin Oral Implants Res. 2017;28(8):902-910. doi:10.1111/clr.12896

9. Vohra F, Al-Kheraif AA, Almas K, Javed K. Comparison of crestal bone loss around dental implants placed in healed sites using flapped and flapless techniques: A systematic review. J Periodontol. 2015;86(2):185-191. doi:10.1902/jop.2014.140355

10. Fugazzotto PA. Treatment options following single-rooted tooth removal: A literature review and proposed hierarchy of treatment selection. J Periodontol. 2005;76(5):821-831. doi:10.1902/jop.2005.76.5.821

11. Paolantonio $M$, Dolci $M$, Scarano A, et al. Immediate implantation in fresh extraction sockets. A controlled clinical and histological study in man. J Periodontol. 2001;72(11):1560-1571. doi:10.1902/ jop.2001.72.11.1560
12. Grassi FR, Grassi R, Rapone B, Alemanno G, Balena A, Kalemaj Z. Dimensional changes of buccal bone plate in immediate implants inserted through open flap, open flap and bone grafting and flapless techniques: A cone-beam computed tomography randomized controlled clinical trial. Clin Oral Implants Res. 2019;30(12):1155-1164. doi:10.1111/clr.13528

13. Juodzbalys G, Sakavicius D, Wang HL. Classification of extraction sockets based upon soft and hard tissue components. J Periodontol. 2008;79(3):413-424. doi:10.1902/jop.2008.070397

14. Monje A, Aranda L, Diaz KT, et al. Impact of maintenance therapy for the prevention of peri-implant diseases: A systematic review and meta-analysis. J Dent Res. 2016;95(4):372-379. doi:10.1177/0022034515622432

15. Furhauser R, Florescu D, Benesch T, et al. Evaluation of soft tissue around single-tooth implant crowns: The pink esthetic score. Clin Oral Implants Res. 2005;16(6):639-644. doi:10.1111/j.1600-0501.2005.01193.x

16. Kadkhodazadeh $M$, Amid R. Evaluation of peri-implant tissue health using a scoring system. JIACD. 2012;4(1):51-57.

17. Cosyn J, Eghbali A, Hermans A, Vervaeke S, De Bruyn H, Cleymaet R. A 5-year prospective study on single immediate implants in the aesthetic zone. JClin Periodontol. 2016;43(8):702-709. doi:10.1111/jcpe.12571

18. Al-Sulaimani AF, Mokeem SA, Anil S. Peri-implant defect augmentation with autogenous bone: A study in beagle dogs. J Oral Implantol. 2013;39(1):30-36. doi:10.1563/aaid-joi-d-10-00132

19. Neves M, Correia A, Alves CC. A novel approach to preserve the buccal wall in immediate implant cases: A clinical report. J Oral Implantol. 2013;39(2):198-205. doi:10.1563/AAID-JOI-D-11-00082

20. Paknejad $M$, Akbari S, Aslroosta $H$, Panjnoush $M$, Hajheidary S. Effect of flapless immediate implantation and filling the buccal gap with xenograft material on the buccal bone level: A randomized clinical trial. J Dent (Tehran). 2017;14(6):344-351.

21. Felice P, Pistilli R, Barausse C, Trullenque-Eriksson A, Esposito M. Immediate non-occlusal loading of immediate post-extractive versus delayed placement of single implants in preserved sockets of the anterior maxilla: 1-year post-loading outcome of a randomised controlled trial. Eur J Oral Implantol. 2015;8(4):361-372.

22. De Bruyn H, Vandeweghe S, Ruyffelaert C, Cosyn J, Sennerby L. Radiographic evaluation of modern oral implants with emphasis on crestal bone level and relevance to peri-implant health. Periodontol 2000. 2013;62(1):256-270. doi:10.1111/prd.12004

23. Calvo-Guirado JL, López-López PJ, Pérez-Albacete Martínez C, et al. Peri-implant bone loss clinical and radiographic evaluation around rough neck and microthread implants: A 5-year study. Clin Oral Implants Res. 2018;29(6):635-643. doi:10.1111/clr.12775

24. Chackartchi T, Romanos GE, Sculean A. Soft tissue-related complications and management around dental implants. Periodontol 2000. 2019;81(1):124-138. doi:10.1111/prd.12287

25. Tarnow DP, Chu SJ, Salama MA, et al. Flapless postextraction socket implant placement in the esthetic zone: Part 1. The effect of bone grafting and/or provisional restoration on facial-palatal ridge dimensional change - a retrospective cohort study. Int J Periodontics Restorative Dent. 2014;34(3):323-331. doi:10.11607/prd.1821

26. Caton JG, Armitage G, Berglundh T, et al. A new classification scheme for periodontal and peri-implant diseases and conditions - introduction and key changes from the 1999 classification. J Clin Periodontol. 2018;45(Suppl 20):S1-S8. doi:10.1111/jcpe.12935

27. Ramanauskaite A, Juodzbalys G, Tözüm TF. Apical/retrograde periimplantitis/implant periapical lesion: Etiology, risk factors, and treatment options: A systematic review. Implant Dent. 2016;25(5):684-697. doi:10.1097/ID.0000000000000424

28. Testori T, Weinstein T, Scutellà F, Wang HL, Zucchelli G. Implant placement in the esthetic area: Criteria for positioning single and multiple implants. Periodontol 2000. 2018;77(1):176-196. doi:10.1111/prd.12211

29. Mombelli A. Maintenance therapy for teeth and implants. Periodontol 2000. 2019;79(1):190-199. doi:10.1111/prd.12255

30. Tomasi C, Donati M, Cecchinato D, Szathvary I, Corrà E, Lindhe J. Effect of socket grafting with deproteinized bone mineral: An RCT on dimensional alterations after 6 months. Clin Oral Implants Res. 2018;29(5):435-442. doi:10.1111/clr.13141 